\title{
Evolution of Complex Filaments
}

\author{
Cristiana Dumitrache
}

Astronomical Institute of Romanian Academy, 75212 Bucharest, Str. Cutitul de Argint 5, Romania

\begin{abstract}
This study focusses on the less studied aspects of filaments evolution: the coupling of two or more filaments to form a complex filament and also the split of one such filament into many components. This phenomenon was first observed by d'Azambuja - we apply statistical mathematical methods to obtain evolutionary sequences and detect the complex filaments.
\end{abstract}

\section{Introduction}

During its life a filament may joint with others and later separate from them, each one following its peculiar evolution. In their work d'Azambuja and d'Azambuja (1948) revealed this kind of phenomenon and called it complex filaments, "des filaments complexes" in French. He also explained the difficulties in studying such phenomena from an observational point of view. It is very difficult to know, after the junction followed by a separation of the filaments, which part of which filament goes in one direction or in another.

By applying a cluster analysis method we can detect, in a computational way, the coupling and splitting of filaments and reveal the aspects linked to photospheric and chromospheric activity. We focus on these less studied aspects of the filament activity. We think that the evolution of a complex filament could be an example of magnetic reconnection and we may also learn new aspects about the prominences' magnetic field.

\section{Data and Method}

A computation based on a cluster analysis method led us to find the evolutionary sequences of filaments (see Dumitrache, Dinulescu and Priest 1994 or Dumitrache 1997) and then to detect the sequences that contain complex filaments. This method of computation strongly depends on the differential rotation laws of filaments. We have used d'Azambuja's law:

$$
\omega=1.22-1.40 \sin ^{2} \phi-1.33 \sin ^{4} \phi
$$

from which we have subtracted the synodical differential rotation $\left(13.2^{\circ} /\right.$ day).

The data on filaments, extracted from the Solnechnyie Dannye Bulletin (1989), are given by the coordinates of their end points (latitude and Carrington longitude). In order to study their evolution, we have also computed for each 
filament the length (the angular distance between the ends), denoted by $y$, and the inclination made with the solar parallel - the tilt angle $x$. This angle is positive for filaments oriented ' $/$ ' and negative for filaments oriented '

We have analyzed many cases of complex filaments for the period 19871989 , taking into account the synoptic maps and the daily observations maps provided by the same catalogue.

\section{Results}

The analysis of particular sequences containing complex filaments has revealed the following general conclusions.

Filaments join as a result of sudden changes of differential rotation velocity: one filament accelerates, while the other decelerates (Figure 1). One filament splits in two or more parts when each part reaches a change of the differential rotation, with very different values of one to the other.

As a continuation of the sudden changes of the differential rotation velocity, sudden changes of the tilt angle $x$ also take place. This could indicate local magnetic reconnections in the photosphere or below.

Many times, when the filaments come together, they undergo sudden disappearances (DB) and, after a period, can reform separately or not at all.

The complex filaments tend to be localized at latitudes between $40^{\circ}$ and $58^{\circ}$. There are very few complex filaments at latitudes less then $40^{\circ}$ and those that are there are linked to the rising of active centers after the filament apparition. The active centers seem to attract the contiguous filaments, producing simultaneously the juncture. After the disappearance of the active center, the complex filament splits again.

There are many complex filaments linked to pivot-point evolutionary sequences. The filaments with pivot points (discovered by Mouradian and SoruEscaut 1989) also attract neighboring filaments to produce complexes.

The polar filaments join together to form one complex, but usually they link to their ends as a longer filament, as their tilt angle decreases in time. After a DB, a long filament may reform as other small separate filaments.

\section{One Particular Case}

We describe here the evolution of four filaments observed in the northern hemisphere, which joined and split in a very complicated process, during their evolution. There are two sequences (denoted S1 and S2) with pivot points, one sequence is the migrator type (S3) and the other is the retrograde type sequence (S4), according to the classification of Dumitrache (1997).

After a sudden disappearance, $\mathrm{S} 1$ formed much later connected to $\mathrm{S} 2$. They evolved together, as one filament, during one rotation and suffered a DB, when the tilt angle $x$ changed in a dramatic way (from negative to positive values). After two rotations, S2 reformed (in rotation no. 1796) and its length elongated very much, being connected with filament $\mathrm{S} 4$ (observed one rotation before). It disappeared again for three days, after which it arose located at two degrees of latitude less then before, together with $\mathrm{S} 1$, as a single huge filament. The 
sequence S3, evolved independently at the beginning, but in rotation 1796 it joined the complex $\mathrm{S} 1+\mathrm{S} 2+\mathrm{S} 4$ in the last day of apparition.

Figure 1 displays the variations of the filaments' differential rotation velocity in time, while Figure 2 shows the variations of the tilt angles, $x$.

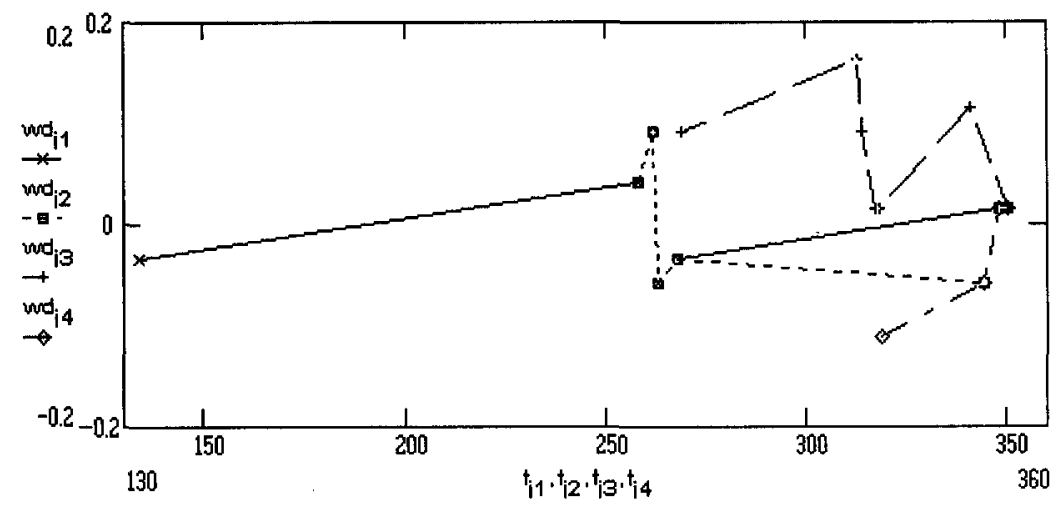

Figure 1. Variation of the differential rotation velocity of filaments with time in days.

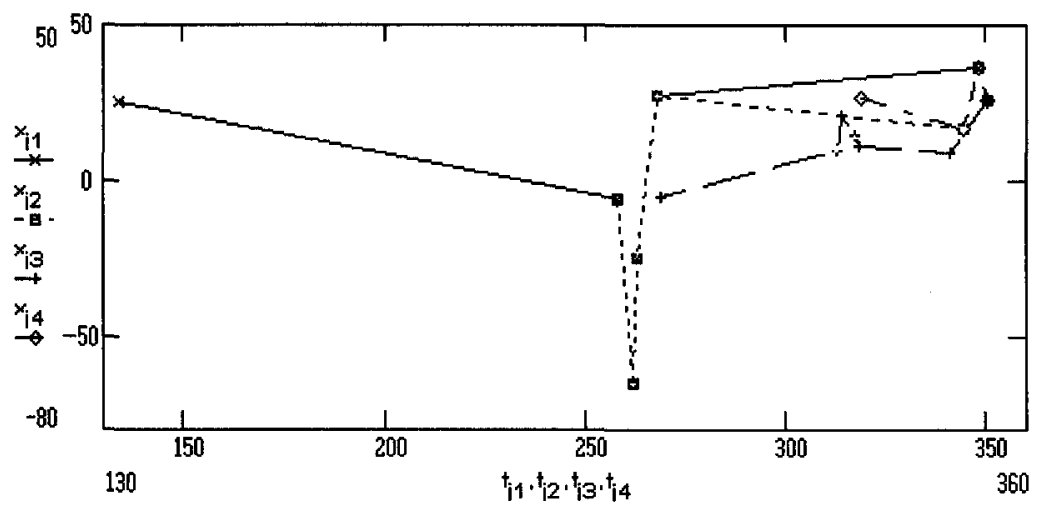

Figure 2. The tilt angle variation with time (days).

The evolution of the filaments' lengths, $y$, is displayed in Figure 3. Figure 4 shows the evolution of the coordinates of these filaments. The filaments' migration in latitude, especially when coupled in a complex, which also have led to the differential velocity changes, are certainly due to the emergence of photospheric magnetic flux. This process is accompanied by magnetic reconnections, indicated also by the change of filament orientations, which have produced DBs.

We suggest that complex filaments are caused by the emergent magnetic flux producing the juncture. The split of filaments may be a consequence of photospheric magnetic field fragmentation. 


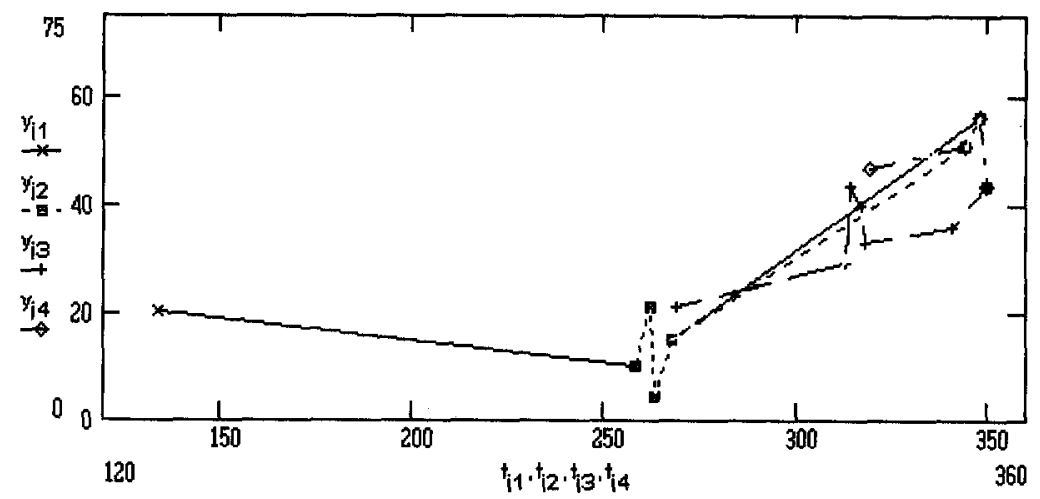

Figure 3. Variation of the filaments' lengths with time (days).

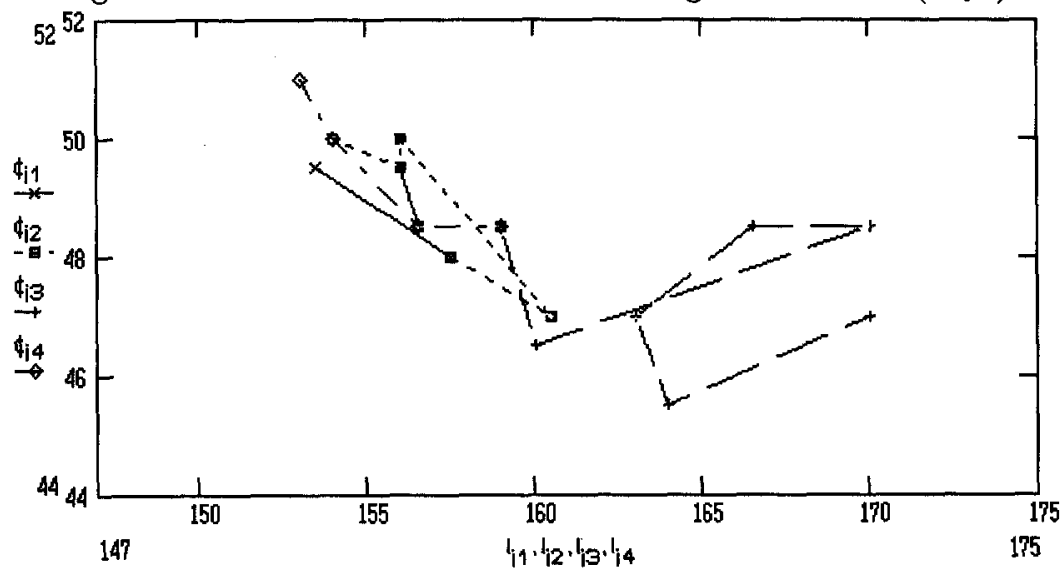

Figure 4. The evolution of the filaments' central coordinates with time (days).

\section{References}

d'Azambuja, L. and d'Azambuja, M. 1948, Ann. Obs. Paris, tome 6, fascicule 7 Dumitrache, C., Dinulescu, S. and Priest, E.R. 1994, in G. Belvedere et al. (eds.), Publ. Obs. Astrof. Catania, Special Volume, p. 65

Dumitrache, C. 1997., Solar Phys., 173, 281

Mouradian, Z. and Soru-Escaut, I. 1989, A\&A, 210, 410

Solnechnyie Dannye Bulletin of the Pulkovo Observatory, 1987-1989 